\title{
Detecting regime transitions in gas-solid fluidized beds from low frequency accelerometry signals
}

\author{
Lilian de Martín $^{\text {a }}$, Javier Villa Briongos ${ }^{\mathrm{b}, *}$, Néstor García-Hernando ${ }^{\mathrm{b}}$, José M. Aragón ${ }^{\mathrm{a}}$ \\ a University Complutense of Madrid, Faculty of Chemistry, Department of Chemical Engineering, Av. Complutense s/n, 28040, Madrid, Spain \\ ${ }^{\mathrm{b}}$ University Carlos III of Madrid, Higher Technical School, Department of Thermal and Fluid Engineering, Av. Universidad 30, 28911, Leganés, Madrid, Spain
}

\begin{abstract}
A B S T R A C T
Low frequency accelerometry signals have been applied for detecting regime transitions in a gas solid fluidized bed. Three solids have been fluidized to promote bubbling, churn and slugging regime. The Kolmogorov entropy and the power spectral density have been used to determine the regime transitions as well as to analyze the dynamical features characterizing the different regimes. Pressure and external acceleration measurements have been taken simultaneously. The accelerometry signal was sampled at $10 \mathrm{kHz}$; then, the envelope was extracted and resampled at $400 \mathrm{~Hz}$. Pressure signal was sampled at $10 \mathrm{kHz}$ and resampled at $400 \mathrm{~Hz}$. Two problems were found during the work: the colored noise present in the envelope and the lack of low frequency information for one of the tested solids. FIR, wavelet and EMD filter strategies have been applied to remove the noise present in the envelope. It is concluded that the envelope of the accelerometry signal might be used to detect regime transition in the same way as the pressure fluctuation signals. Both Kolmogorov and spectral analysis exhibit common features to those obtained from pressure signal analysis, supporting the hypothesis of using low frequency accelerometry instead of conventional pressure measurements for monitoring fluidized beds.
\end{abstract}

\section{Introduction}

The monitoring of gas solid fluidized beds (FB) is an important issue. As the dynamical behaviour characterizing these systems is complex, it is necessary to monitor different bed properties as continuously as possible. As an example of that dynamical complexity, it has been shown that, depending on the operational condition, FB can exhibit dynamical chaotic features [1,2], which can be used to predict malfunctioning [3,4]. Besides the large research effort towards developing measurement techniques aimed to get knowledge on the dynamic phenomena that take place within the bed $[5,6]$ the pressure fluctuations measurements remain as the most used technique $[7,8]$. Its low cost and the direct relation which exists between the property measured and the bed dynamics make up for its disadvantages. Among them, it is worth mentioning its intrusively or local characteristics of the pressure measurement. Moreover, the pressure probes can represent an obstacle for the free development of bubbles and solid motion, affecting the measured dynamics; also, solid probe blockage might appear.

According to that, the need for non invasive measurement methods is clear. These methods can offer several advantages with respect to the invasive methods. Thus, because they are located

\footnotetext{
* Corresponding author. Tel.: + 34 916248392; fax: + 34916248810 E-mail address: jvilla@ing.uc3m.es (J.V. Briongos).
}

outside the vessel, they neither suffer the harsh operating conditions inside the bed nor interfere with the bed dynamics. Nevertheless, the application of these methods in industrial fluidized beds is still an unsolved matter; some factors, such as the large size of industrial facilities, become a big challenge for reported non invasive measure ment techniques such as tomography, ECT.

During the last decade, the need for non invasive techniques has led to the development of acoustic monitoring methods based on microphone, hydrophone and accelerometer measurements [9 16]. Most of these works use a very high sampling frequency, which leads to a large amount of data and limits the use of advanced techniques (like phase space analysis) due to the high computational cost required. To the best of the authors' knowledge, there are few works in literature which use advanced techniques over external passive acoustic signals for getting low frequency information $[10,15,16]$, being the sensibility of the acoustic sensors a limitation for collecting some useful information.

The poor signal/noise ratio obtained in [15] led to the use of new high sensibility acoustic sensors in order to obtain a signal with better quality. In [17] was reported that by using high sensitivity accelerometers is possible to get a low frequency signal related to bulk and bubble dynamics. In that work, the analysis was made over the envelope of the accelerometry signal and not over the direct accelerometry signal. Those promising results led to the necessity of further research on the possibility of using low frequency accel erometry for bed monitoring and dynamic diagnosis purposes. 
According to that, this work focuses on the applicability of the envelope signal for detecting transitions between fluidization regimes. With this purpose, three different solids were fluidized under several air reduced velocities in order to induce different fluidization regimes (bubbling, churn and slugging). Then, by monitoring the evolution of the Kolmogorov entropy of the pressure signal with the reduced air velocity is possible to detect the regime transitions [18].

Following the methodology reported in de Martín et al. [17], the results obtained from the pressure sensor have been compared with those obtained from the accelerometer, which are directly related with the conventional pressure fluctuation measurements [17]. Moreover, the power spectral density of the accelerometry signal for several regimes is shown in order to see the relation between the frequency content with the fluidization dynamics. Furthermore, the main operational problems found during the work were the presence of high level noise in the envelope signal, and the lack of low frequency information for one of the tested solids.

\section{Analysis techniques}

\subsection{Envelope extraction and the Hilbert transform}

In this work, we are going to study the envelope of the accelerometry signal instead the direct accelerometry signal [17]. The reason to do this, is that the envelope signal is more related to the forces that take place inside the bed than the direct signal, which is corrupted by the vessel dynamics and, consequently, it is strongly dependent on the natural frequencies, size, material, geometry, supports etc. of the facility [19].

The hypothesis of using the envelope signal instead the direct acceleormetry signal for fluidized bed dynamics characterization comes from the random vibration theory [20], where it is stablished that a random process can have embedded or encoded two new processes as phase and/or envelope [21 23]. It is worth to mention that to have a useful envelope, the slow varying envelope conditions must be satisfied for the measured accelerometer signals [21].

Accordingly, the envelope of the accelerometry signal was extracted by means of the Hilbert transform. Despite there being many ways of extracting the envelope of a signal, those based on the Hilbert transform (Eq. (1)) have the advantage of providing an analytic expression for the envelope $A(t)$ of the accelerometry signal $a(t)[24,25]$.

$b(t)=\frac{1}{\pi} \int_{\infty}^{\infty} \frac{a\left(t^{\prime}\right)}{t-t^{\prime}} d t^{\prime}$

Where de principal value of the integral is always used. Then, considering $\mathrm{a}(\mathrm{t})$ as a non stationary dynamical process having evolutionary spectral density [20], its envelope is given by

$A(t)=\sqrt{ } a(t)^{2}+b(t)^{2}$

And the instantaneous phase angle can be computed by

$\varphi(t)=\arctan \left(\frac{b(t)}{a(t)}\right)$

Where $a(t)$ and $b(t)$ are orthogonal non stationary dynamical processes defined by an analytic signal of the form $Y(t)=a(t)+b(t) i$.

\subsection{Frequency domain}

The frequency domain analysis has been widely applied to characterize fluidization regimes $[18,26]$ and to get knowledge on the physical phenomena that take place inside a fluidized bed $[27,28]$.
Among the different spectral properties, the power spectrum density (PSD) provides a picture of the characteristic frequencies appearing within a signal. There are different methods to estimate the spectra; here, Welch's method is used for the PSD estimation by averaging 23 periodograms, achieving a good trade off between resolution and error [29].

\subsection{Kolmogorov entropy}

Several methods have been proposed for estimating the Kolmo gorov entropy from experimental time series [30,31]. However, due to the non linear features of the signals collected from FB's [32], the maximum likelihood method [31], which has lower noise influence, is commonly used. For computing the Kolmogorov entropy, the attractor has been reconstructed following the recommendations of Schouten et al. [33]. Accordingly, the average cycle time is used in the present contribution as "orbital time". Finally, a time step of 1 has been used during the reconstruction of the attractor. In order to guarantee that most of the attractor structure is investigated, the Kolmogorov entropy is calculated four times for each attractor, averaging the results.

\subsection{Filters}

The accelerometry envelopes and the pressure signals were filtered before the estimation of the Kolmogorov entropy. The pressure signals were filtered with a low pass Chebyshev filter with a cut off frequency of $20 \mathrm{~Hz}$. The accelerometry envelopes were filtered with three different filters in order to see the influence of the filtering in the Kolmogorov values: A low pass FIR filter, a wavelet filter and a EMD based filter. The choice of the cut off frequency is detailed in the Appendix A.

\subsubsection{Finite Impulse Response (FIR) filter}

The linear low pass or band pass filters are broadly applied in literature for conditioning the pressure signal under investigation $[18,34]$. Broomhead et al. [35] have shown that the Finite Impulse Response (FIR) filters are more suitable for the conditioning of chaotic signals than the Infinite Impulse Response (IIR) filters. In this paper, a low pass FIR filter with a cut off frequency of $20 \mathrm{~Hz}$ is used (Appendix A). The FIR filter has been designed following the method proposed by Parks and McClellan [36].

\subsubsection{Wavelet filter}

The objective of the wavelet filter is to suppress the noise part of the signal $\mathrm{x}(\mathrm{n})$ by discarding the noise $\mathrm{e}(\mathrm{n})$ and to recover the signal of interest $f(n)$. The model for the noisy signal $x(n)$ is basically of the following form:

$x(n)=f(n)+\sigma e(n)$

The de noising procedure includes a decomposition, thresholding detail coefficients and reconstruction of the signal [37]: The Daubechies wavelet (db15) [38] is used in this work since it has a high number of vanishing moments for a given support width, ensuring that the signal analysis becomes more precise with the higher order of polynomials used for approximation. Also, the high regularity of this wavelet is useful for getting nice features of the reconstructed signal, like smoothness [37]. The decomposition level chosen is 4 . Since the noise level is high, the thresholding method used is the universal (Eq. (5)). Such a threshold guarantees with high probability that all the components attributed to noise will have lower amplitudes [39].

$T H R=\sigma \sqrt{2} \log (N)$ 
Where $\mathrm{N}$ is the length of the time series. Since the noise present in the envelope $e(n)$ is colored (see Appendix A), the basic model (Eq. (4)) needs to be adapted by carrying out a level dependent estimation of the level noise [37]. This filter has a quick implemen tation and it does not need a cut off frequency as input parameter, being this fact an advantage over the FIR an EMD filters used in this work.

\subsubsection{Empirical Mode Decomposition filter}

The essence of the empirical mode decomposition (EMD) is to identify the intrinsic mode functions (IMF) embedded within a time series, which belong to different time scales dictated by the time lapse between the extremes, and to sift them out to obtain one intrinsic mode at a time. The decomposition has been explained in detail in literature $[40,41]$. Once the time series has been decomposed into a finite number M of IMFs $C_{k}(t)$, associated with various time scales and the residual $r(t)$, the time series can be reconstructed by superposi tion of the IMFs and the residual:

$x(t)=\sum_{k=1}^{M} C_{k}(t)+r(t)$

The major advantage of the EMD is that the basic functions are derived from the signal itself. Hence, the analysis is adaptive in contrast to the wavelet method, where the basis functions are fixed. The instantaneous frequencies of an IMF $\mathrm{k}$, can be computed by calculating its Hilbert transform (Eq. (1)) and differentiating the phase angle (Eq. (3)).

$\omega_{k}(t)=\frac{d \varphi_{k}(t)}{d t}$

The cut off frequency is $20 \mathrm{~Hz}$ (Appendix A). Accordingly, the filtered signal has been reconstructed by adding only that IMFs with average instantaneous frequency $\bar{\omega}_{k}$ lower than $20 \mathrm{~Hz}$, where $\bar{\omega}_{k}$ is:

$\bar{\omega}_{k}=\frac{1}{N} \sum_{n=1}^{N} \omega_{k}(n)$

$\mathrm{n}$ are the instants where the instantaneous frequency is calculated and $\mathrm{N}$ is the length of the time series. This filter performs better nonlinear filtering than the wavelet one [42], being capable of preserving the local features appearing in the signal.

\section{Experimental}

Accelerometry and pressure fluctuation signals were recorded in a cylindrical poly methyl methacrylate (PMMA) vessel $0.07 \mathrm{~m}$ ID. The air distributor consists of 7 tuyeres that provide a homogeneous air distribution. Acceleration and pressure signals were measured simultaneously during 4 minutes. Regarging to the sampling, for the pressure signal a sampling frequency of $400 \mathrm{~Hz}$ is enough to carry out the phase space analysis. Nevertheless, the accelerometry signal needs a higher sampling frequency to capture the high frequency vibrations with the lowfrequency envelope associated. To avoid resonant amplification in the accelerometer, the sampling frequency of the accelerometry signal has been set at $10 \mathrm{kHz}$. Since the data adquisition hardware used does not allow choosing different sampling frequencies for the different modules (pressure and acceleration), the initial sampling frequency chosen for all the signal was $10 \mathrm{kHz}$. Later, the envelope of the accelerometer signal was estimated by means of the Hilbert transform (Eq. (1)). Since the envelope and the pressure signal contain low frequency information, they have been resampled to $400 \mathrm{~Hz}$ (Fig. 1). The ratio $\mathrm{H}_{\mathrm{mf}} / \mathrm{D}$ was fitted approximately to 2 in all the runs. The pressure probe and the accelerometer are located at $0.125 \mathrm{~m}$ from the distributor. The accelerometer was placed on the outside vessel wall, as close as possible to the pressure probe.

\subsection{Acceleration measurement system}

The horizontal vibrations of the vessel wall were collected by means of a commercial accelerometer (Brüel and Kjaer Type 4507 B 005) located on the external face of the vessel wall by means of a mounting slot supported with glue. The accelerometer is fitted to a NI 9233 (National Instruments) acquisition module, which is inserted in a chassis NI cDAQ 9172 connected to a PC. The software used to acquire the signals is Labview 8.5. The sensor has a reference sensitivity of $\sim 1 \mathrm{~V} / \mathrm{g}$ and a resonant frequency of $16.3 \mathrm{kHz}$. Special care was taken with the wire that connects each accelerometer with the data acquisition device to avoid mechanical and triboelectric noise in the signal.

\subsection{Pressure measurement system}

Pressure fluctuations were recorded by a probe connected to a pressure transmitter PR3110 (Ellison Sensors) fitted to a NI 9205 data acquisition module. This device was also mounted in the chassis cDAQ 9172. The distance between the pressure probe and the transmitter is around $0.1 \mathrm{~m}$.

\subsection{Solids}

Three different solids were fluidized to study different fluidization regimes and transitions, and to see the influence of the particles properties on the characteristics of the accelerometry signal. The characteristics of the fluidized bed and particles are shown in Table 1.

The range of the reduced air velocity used is also shown in the table. Despite the fact that the fluidization of ballotini glass beads has no industrial application, this solid is used in this work because high energy vibrations are expected due to the hardness of the particles. Olivine and alperujo were also fluidized. Olivine is commonly used as catalyst in bed tar elimination in biomass gasifiers [43]. Alperujo (also called olive kernels), which is a by product of olive oil extraction, is usually gasified in FB's [44]. Both olivine and alperujo are particles softer than the ballotini glass beads, accordingly it is expected vibrations less energetic for the olivine and alperujo fluidization than for the ballotini fluidization.

\subsection{Operation fluidization regimes}

The different solids and air velocities used have led to the appearance of different fluidization regimes. These states are qualitatively described below:

- Bubbling regime: As described previously in literature [7], several bubbles simultaneously present within the bed that increase their size quickly with the distance from the distributor; as a result, the bubble distributions (bubble number and size) are different in the lower and upper parts of the bed. Moreover, it has been observed that the bed geometry (narrow bed) strongly influences the resulting PSD, the narrow bed feature of the facility used here makes the natural bed frequency dominate the spectra.

- Churn type regime: There is some controversy about the existence of this regime in gas solid FB [45]. In this work, we refer to a form of developing slug flow in short pipes with the appearance of a churning motion. Consequently, that region of churn like flow is characterized by large bubbles with a diameter similar to the vessel size which cause a vigorous fluidization process.

- Slugging regime: In slugging regime [10], each rising bubble spans nearly up to the entire cross section of the bed and pushes a large mass of particles in front of it. Each ascending gas pocket is referred 

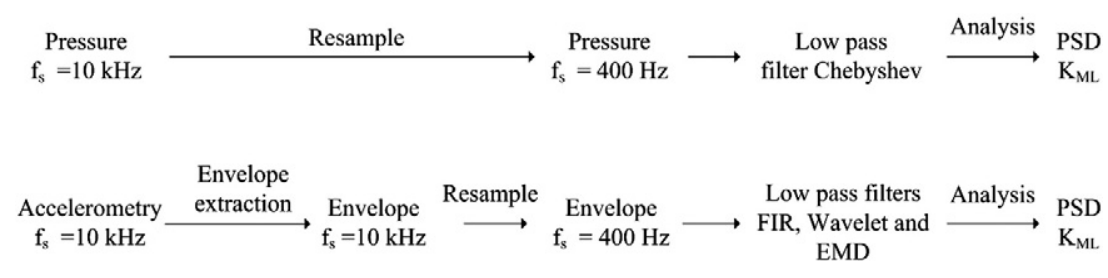

Fig. 1. Scheme of the methodology followed.

to as a slug. Particles move downward through and around the rising slug until it reaches the top of the bed, whereupon a settled bed is re established, and the cycle repeats. As the fluidizing velocity approaches a critical value, slugging becomes nearly periodic and appears to be very regular.

\section{Results}

\subsection{Ballotini particles: From bubbling regime to churn regime}

By monitoring the evolution of the Kolmogorov entropy $\left(\mathrm{K}_{\mathrm{ML}}\right)$ of the pressure signal (Fig. 2), a regime transition is detected around $\mathrm{u}_{\mathrm{r}} \sim 1.8$, since the tendency of $\mathrm{K}_{\mathrm{ML}}$ changes abruptly around this reduced velocity. The entropy evolution observed in Fig. 2 is similar to that shown in [18] for captive conditions, with the presence of a maximum at low air velocities. Thus, a transition from bubbling regime to churn regime is visually confirmed around $\mathrm{u}_{\mathrm{r}} \sim 1.8$.

To see the spectral characteristics of the different regimes, the pressure PSD were calculated for $\mathrm{u}_{\mathrm{r}}=1.2$ and $\mathrm{u}_{\mathrm{r}}=3.0$ (Fig. 3). The frequency distribution is similar for both regimes, indicating the presence of simultaneous bubbles in the bed [7]. Nevertheless, it can be observed how both spectra exhibit some different frequency content between 5 to $10 \mathrm{~Hz}$. Thus, the $510 \mathrm{~Hz}$ region is wider for $\mathrm{u}_{\mathrm{r}}=1.2$ (Fig. 3a) than for $\mathrm{u}_{\mathrm{r}}=3.0$ (Fig. 3b). However, due to the high similarity between the spectra, the use of the Kolmogorov entropy is necessary to detect the transition regime.

In the bubbling regime, the high number of bubbles coexisting and interacting between them in the bed leads to a complex dynamic with high Kolmogorov entropy values (Fig. 2). When the air flow is increased and the churn regime is established, only a few large bubbles are present simultaneously in the bed, the number of bubble interactions is lower than in the bubbling case, and the Kolmogorov entropy decreases. It was observed that the trend of the Kolmogorov entropy changes again around $\mathrm{u}_{\mathrm{r}} \sim 3.5$. This is probably due a transition to intermediate turbulent regime [18]. Nevertheless, it is not possible to state this conclusion because of the lack of data at higher velocities.

The behaviour of the Kolmogorov entropy calculated from the accelerometry signal is shown in Fig. 4. The evolution of $\mathrm{K}_{\mathrm{ML}}$ for the three filters is similar to that obtained from the pressure signal, detecting a regime transition around $\mathrm{u}_{\mathrm{r}} \sim 1.8$. All the filters provide good results, but the entropy of the signal filtered with the EMD filter shows a smoother behaviour. This makes possible to detect the transitions more accurately with the EMD filter than with the wavelet or FIR.

The spectra of the accelerometry signal for bubbling regime (Fig. 5a) and for churn regime (Fig. 5b) also reveal clear differences

Table 1

Bed characteristics and operational range of the reduced air velocity $\left(\mathrm{u}_{\mathrm{r}}(-)\right)$.

\begin{tabular}{llllll}
\hline Solid & $\mathrm{d}_{\mathrm{p}}(\mathrm{m})$ & $\rho_{\mathrm{p}}\left(\mathrm{kg} / \mathrm{m}^{3}\right)$ & $\mathrm{u}_{\mathrm{mf}}(\mathrm{m} / \mathrm{s})$ & Geldart & $\mathrm{u}_{\mathrm{r}}(-)$ \\
\hline Ballotini & $3.8 \mathrm{E}-4$ & 2464 & 0.11 & $\mathrm{~B}$ & $1.2-3.7$ \\
Olivine & $1.2 \mathrm{E}-3$ & 2793 & 0.67 & $\mathrm{D}$ & $1.2-2.3$ \\
Alperujo & $4.0 \mathrm{E}-4$ & 961 & 0.09 & $B$ & $1.4-3.3$ \\
\hline
\end{tabular}

between both fluidization states. It is worth pointing out that in contrast to pressure fluctuation time series, those differences take place below $2 \mathrm{~Hz}$ and not in the 5 to $10 \mathrm{~Hz}$ frequency band.

\subsection{Olivine particles: From churn regime to slugging regime}

The evolution of the Kolmogorov entropy of the pressure signal (Fig. 6) for olivine particles shows a regime transition around $\mathrm{u}_{\mathrm{r}} \sim 1.3$. The $\mathrm{K}_{\mathrm{ML}}$ decreases quickly from $\sim 30 \mathrm{bit} / \mathrm{s}$ to $\sim 7 \mathrm{bit} / \mathrm{s}$, where it stays constant. As expected, a maximum in the entropy is not observed in this operational range. This lack of maximum is related with the fluidization characteristics of Geldart D particles. For D particles, the bubbling/churn regime only exists over a narrow flow range, changing abruptly to slugging at higher flow rates [10].

As for the ballotini case, in order to study the spectral character istics of the regimes that take place in the bed, the pressure spectra for $\mathrm{u}_{\mathrm{r}}=1.2$ and $\mathrm{u}_{\mathrm{r}}=2.1$ are shown in Fig. 7. The spectrum and the entropy values for $\mathrm{u}_{\mathrm{r}}=1.2$ (Fig. 7a) are qualitatively similar to those previously observed for the churn regime of the ballotini particles (Fig. 3b). On the contrary, the spectrum for $\mathrm{u}_{\mathrm{r}}=2.1$ (Fig. 7b) reveals a high persistence of the pressure signal. This high periodicity is characteristic of the slugging regime and is reflected in the low values of the Kolmogorov entropy found at these reduced velocities (Fig. 6). Moreover, visual observation of the flow confirms a transition between churn and slugging regimes. Therefore, from these results of the frequency and state space analysis, it is concluded that the transition takes place around $\mathrm{u}_{\mathrm{r}} \sim 1.2$.

By monitoring the evolution of the Kolmogorov entropy of the accelerometry signals (Fig. 8), the same conclusion can be inferred from the envelopes filtered with the wavelet and EMD filters. For these two filters, the entropy decreases quickly from $\sim 60 \mathrm{bit} / \mathrm{s}$ to $~ 35$ $\mathrm{bit} / \mathrm{s}$ and then decreases slower when the slugging regime is established.

The entropy of the envelope signal filtered with the FIR filter also decreases quickly in the regime transition zone, but the behaviour is

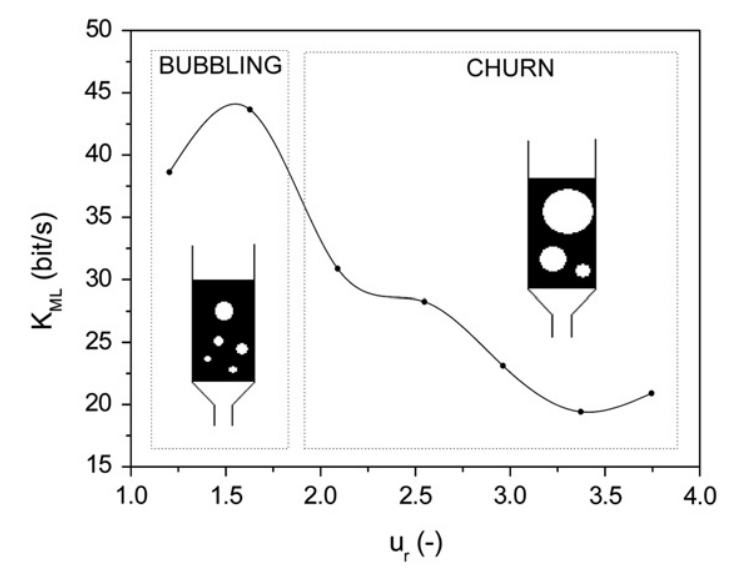

Fig. 2. Kolmogorov entropy vs. reduced air velocity for the pressure signal. Ballotini particles (Geldart B). 

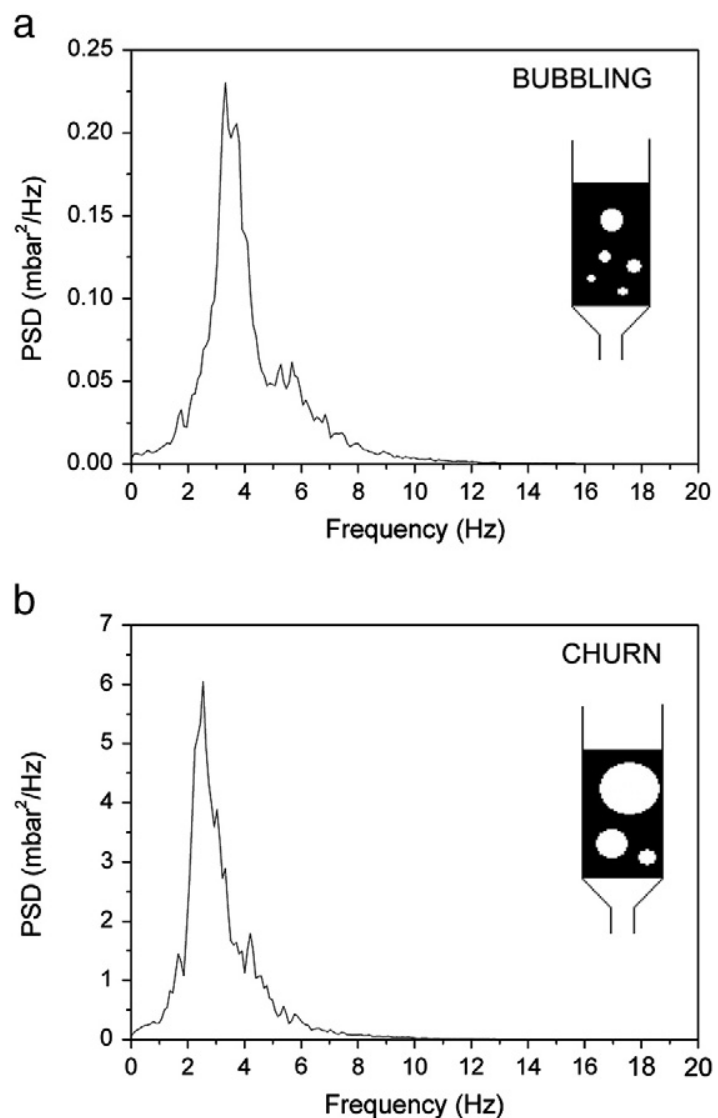

Fig. 3. PSD of the pressure signal for Ballotini particles (Geldart B). (a) Bubbling regime $\mathrm{u}_{\mathrm{r}}=1.2$, (b) Churn regime $\mathrm{u}_{\mathrm{r}}=3.0$.

not as clear as the one observed with the data filtered with wavelet and EMD filters.

As for the pressure fluctuation signals, the PSD also reflect differences between the regimes (Fig. 9).

\subsection{Alperujo particles: From bubbling regime to churn regime}

The behaviour of the Kolmogorov entropy of the pressure signal is shown in Fig. 10; in this case the regime transition takes place around $\mathrm{u}_{\mathrm{r}} \sim 1.5$. It can be see that the evolution of this invariant is similar to

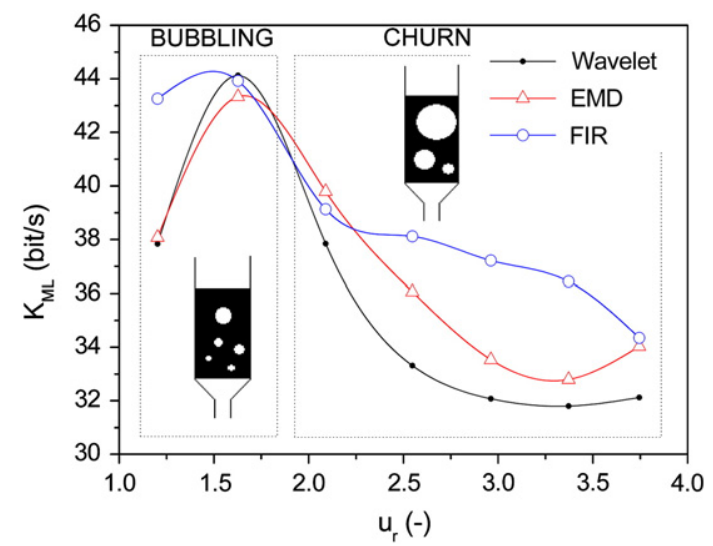

Fig. 4. Kolmogorov entropy vs. reduced air velocity for the accelerometry signal. Ballotini particles (Geldart B).
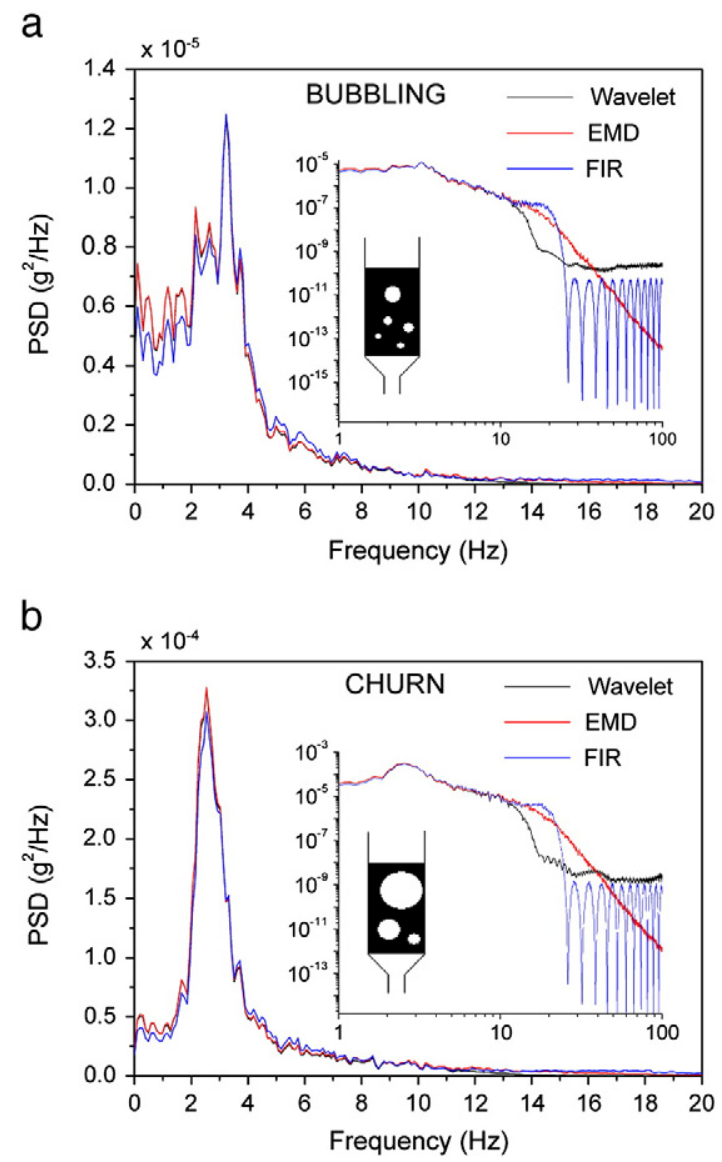

Fig. 5. PSD of the accelerometry signal for Ballotini particles (Geldart B). (a) Bubbling regime $\mathrm{u}_{\mathrm{r}}=1.2$, (b) Churn regime $\mathrm{u}_{\mathrm{r}}=3.0$.

that shown for ballotini particles (Fig. 2) except for the lack of maximum. This lack of maximum, which is associated to the instability of the bubble regime (the same that the olivine case) is explained focusing on the nature of the alperujo solid.

This solid is obtained from the olive kernels and is composed by two well differentiated types of particles (both of them belong to the same sieving fraction and with a medium size of $4.0 \mathrm{E} 4 \mathrm{~m}$ ). One type of particles is formed by the olive's stone and the other one by the

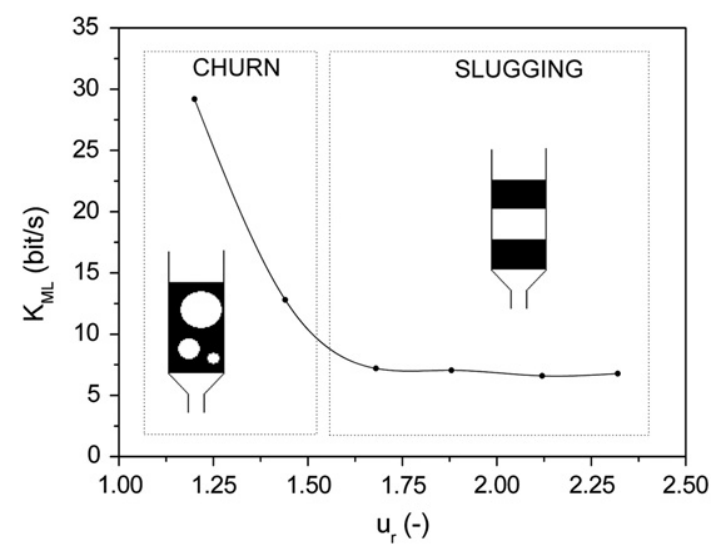

Fig. 6. Kolmogorov entropy vs. reduced air velocity for the pressure signal. Olivine particles (Geldart D). 

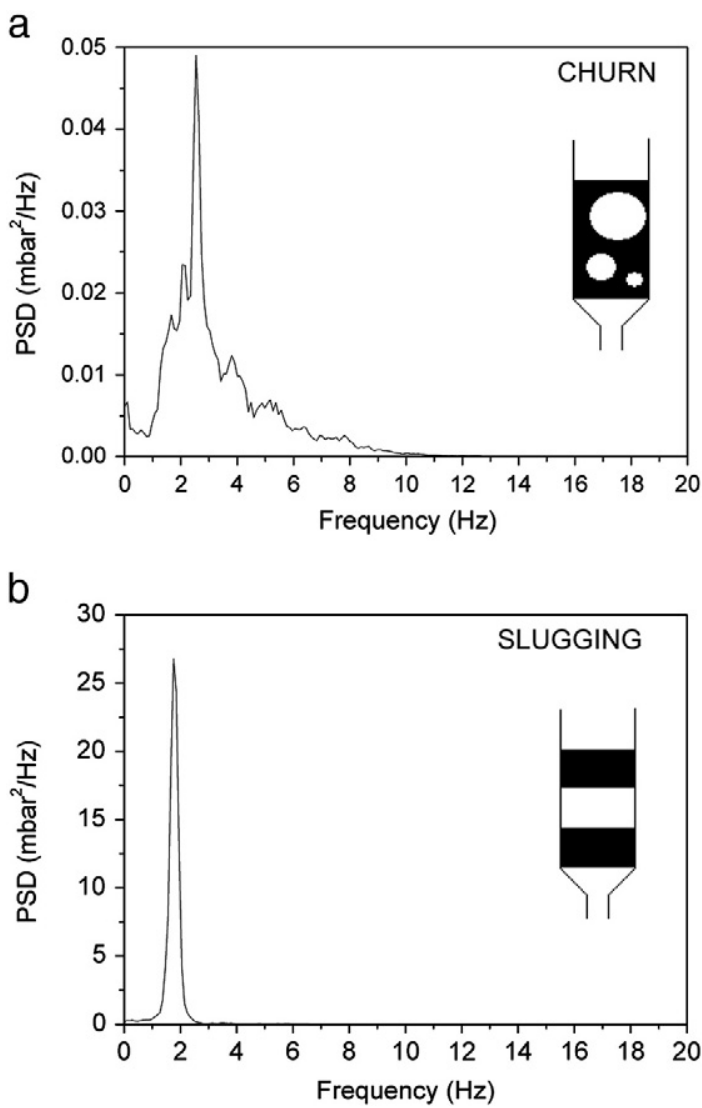

Fig. 7. PSD of the pressure signal for olivine particles (Geldart D). (a) Churn regime $\mathrm{u}_{\mathrm{r}}=1.2$, (b) Slugging regime $\mathrm{u}_{\mathrm{r}}=2.1$.

pulp. The stone and the pulp have very different fluid dynamic properties due to the differences in the density and, specially, due to the differences in their sphericity (the pulp particles are similar to small needles and the stone particles are rounder). This mixture of particles provides the alperujo of a behavior more similar to the $\mathrm{D}$ Geldart solids than to the B Geldart solids (even though it belongs to the B classification). A complete description of the alperujo fluid dynamics can be found in [46].

The spectrum of the pressure signal for the first regime $u_{r}=1.4$ (Fig. 11a) is complex, since it is characterized by a uniform frequency band from 2 to $6 \mathrm{~Hz}$, revealing the presence of multiple bubbles in the bed. In contrast, the spectrum of the second regime $u_{r}=2.7$ (Fig. 11b) exhibits a maximum frequency around $3 \mathrm{~Hz}$. This value can be

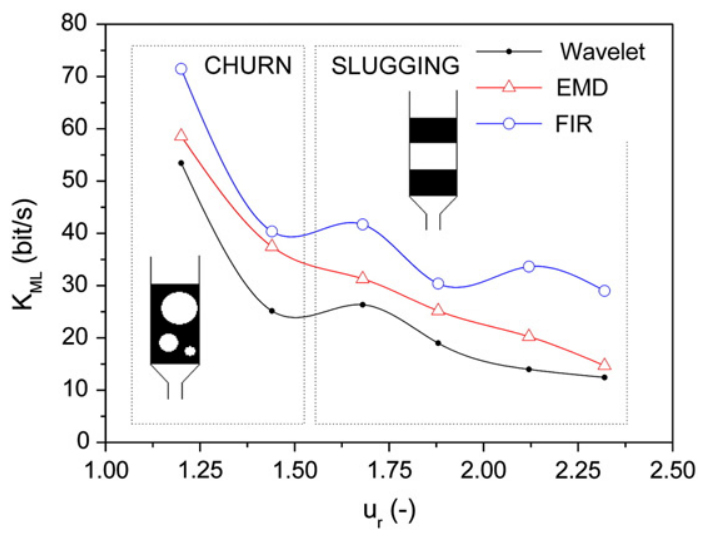

Fig. 8. Kolmogorov entropy vs. reduced air velocity for the accelerometry signal. Olivine particles (Geldart D).
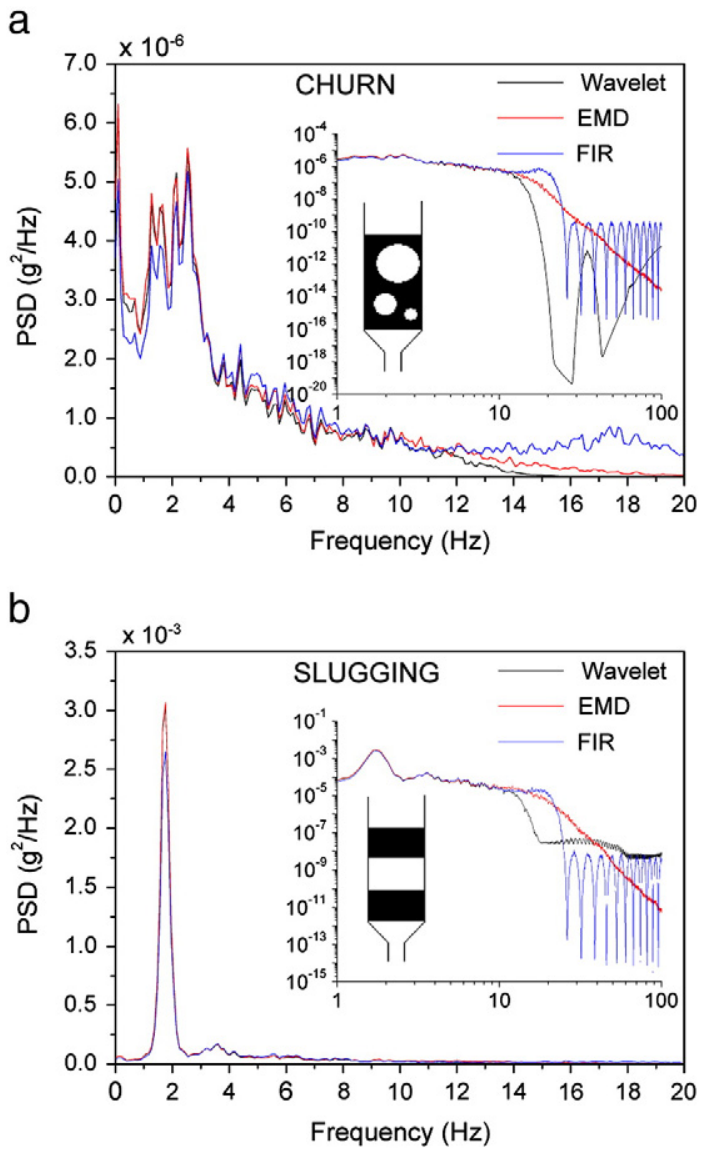

Fig. 9. PSD of the accelerometry signal for olivine particles (Geldart D). (a) Churn regime $\mathrm{u}_{\mathrm{r}}=1.2$, (b) Slugging regime $\mathrm{u}_{\mathrm{r}}=2.1$.

explained by the natural frequency of the bed, $\mathrm{f}_{\mathrm{n}}=2.7 \mathrm{~Hz}$ [47], whose component within the pressure signal gains importance due to the churning nature of the flow. These results together with the visual inspection of the fluidization state reveal that the transition observed around $\mathrm{u}_{\mathrm{r}} \sim 1.5$ takes place from bubbling to churn regime. It is worth pointing out that the spectrum of alperujo for bubbling regime is more complex than the spectrum of ballotini for the same regime. The different solid properties, such as the low density of the alperujo particles $\left(961 \mathrm{~kg} / \mathrm{m}^{3}\right)$ compared with the ballotini particles $(2464 \mathrm{~kg} /$ $\mathrm{m}^{3}$ ) and the sphericity, lead to differences in the number and size of bubbles present in the bed for the same bubbling regime. This is also

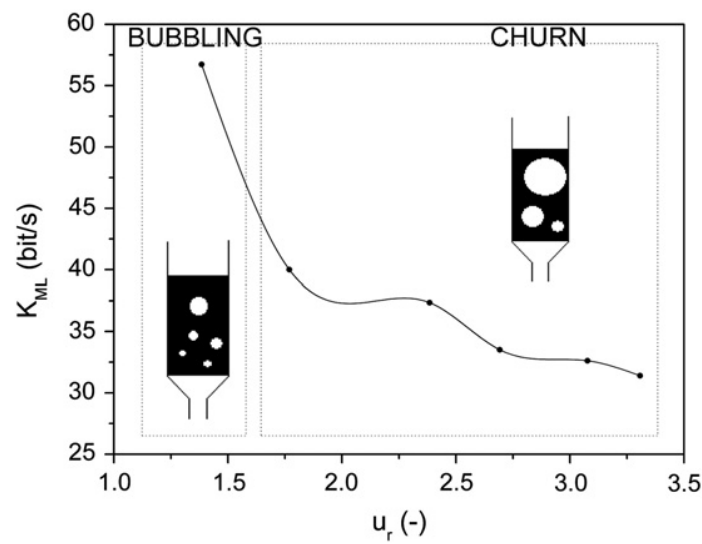

Fig. 10. Kolmogorov entropy vs. reduced air velocity for the pressure signal. Alperujo particles (Geldart B). 
a

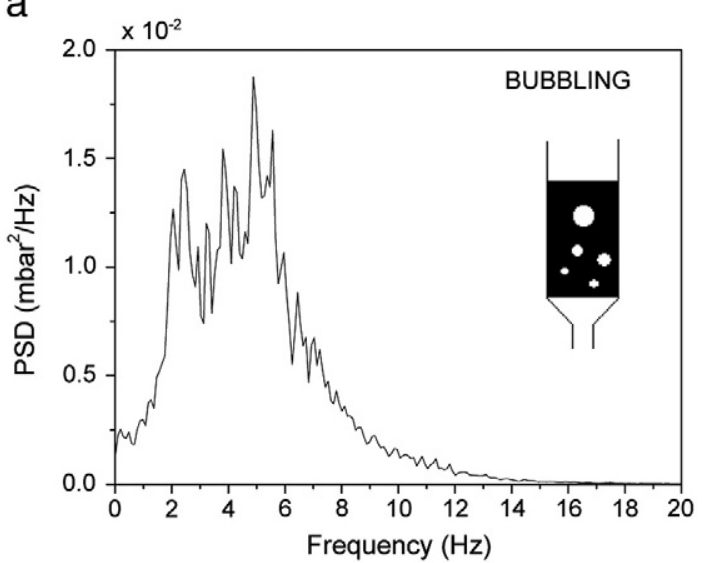

b

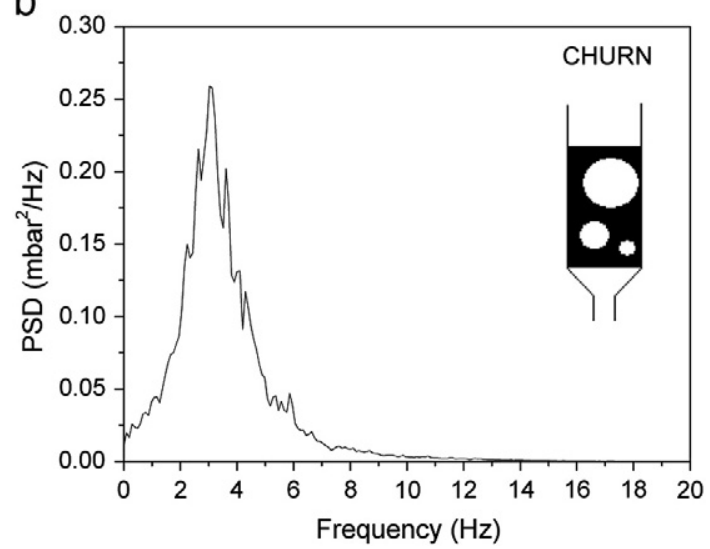

Fig. 11. PSD of the pressure signal for alperujo particles (Geldart B). (a) Bubbling regime $\mathrm{u}_{\mathrm{r}}=1.4$, (b) Churn regime $\mathrm{u}_{\mathrm{r}}=2.7$.

revealed by the Kolmogorov entropy, which is higher for alperujo ( $\sim 57 \mathrm{bit} / \mathrm{s})$ than for ballotini $(\sim 44 \mathrm{bit} / \mathrm{s})$ for the same regime.

The Kolmogorov entropy of the accelerometry signal is shown in (Fig. 12). The values for $\mathrm{u}_{\mathrm{r}}=1.4$ and $\mathrm{u}_{\mathrm{r}}=1.6$ are not represented in this figure because low frequency information was not obtained for these low air velocities. Probably the low density of the particles together with the softness of the material and the low air velocity lead to poor signal emissions. Although the sensitivity and range of the accelerometer is high, it might not be high enough to detect changes

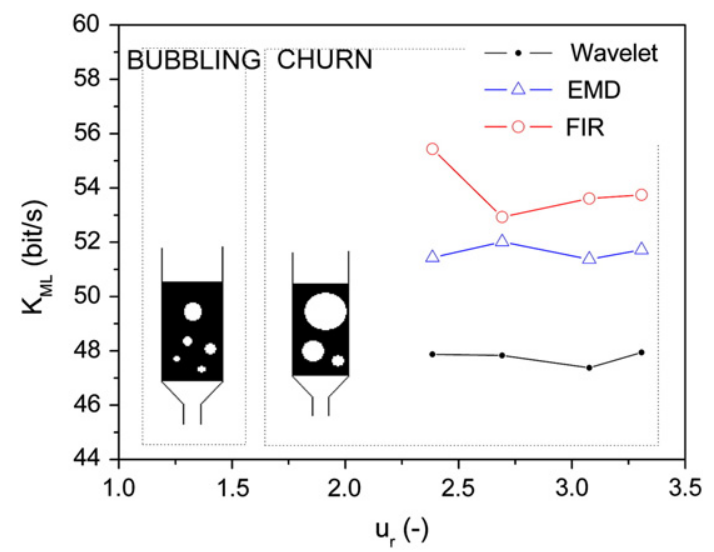

Fig. 12. Kolmogorov entropy vs. reduced air velocity for the accelerometry signal. Alperujo particles (Geldart B). a

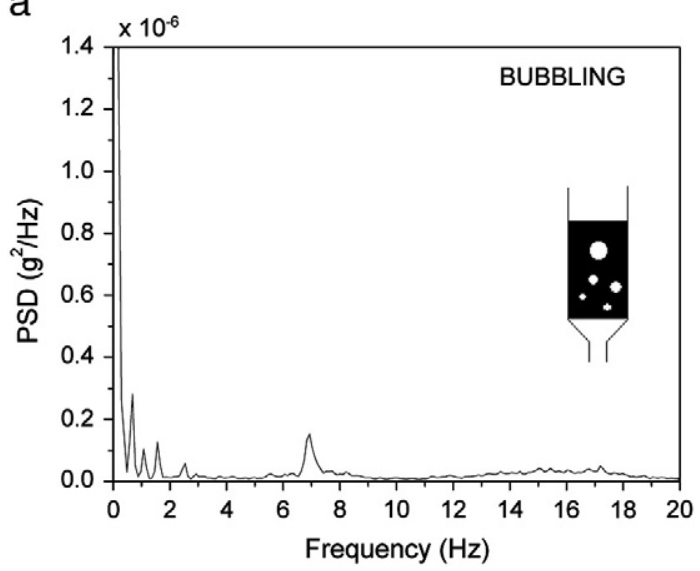

b

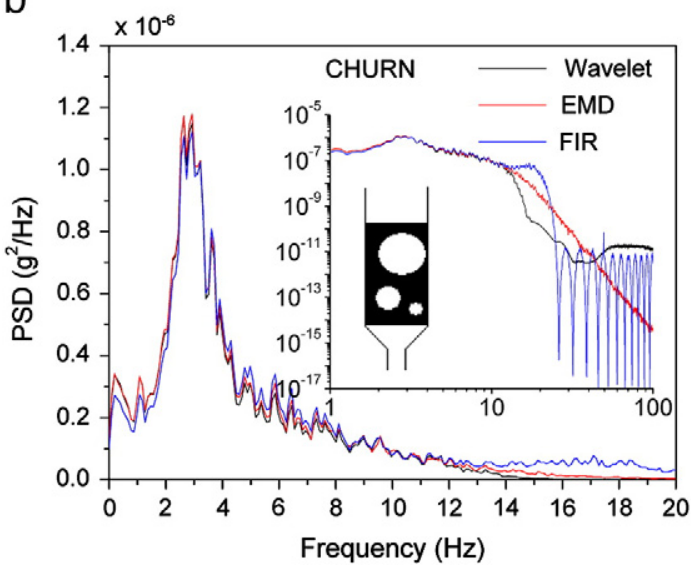

Fig. 13. PSD of the accelerometry signal for alperujo particles (Geldart B). (a) Bubbling regime $\mathrm{u}_{\mathrm{r}}=1.4$, (b) Churn regime $\mathrm{u}_{\mathrm{r}}=2.7$.

in the energy of the vibrations at these air velocities. The lack of low frequency information is well observed in the spectrum for $\mathrm{u}_{\mathrm{r}}=1.4$ (Fig. 13a). The spectrum of the envelope is similar to the spectrum of the direct signal, without the presence of low frequencies. Later, as the air velocity raises fluidized bed dynamical information can be recovered again from the envelope (Fig. 13b).

\section{Conclusions}

Under certain conditions, the envelope of the accelerometry signal sampled at $400 \mathrm{~Hz}$ can be used to accurately determine regime transitions in lab scale gas solid fluidized bed. These results show the possibility of using accelerometers instead of conventional pressure transducers for monitoring and dynamic diagnosis purposes.

The spectrum and the Kolmogorov entropy of the filtered envelope and the pressure signal detect the fluidization regime transition in a similar way.

The noise present in the envelope has to be removed with a suitable filter, in order to recover the exponential fall off of the signal. As expected, the wavelet and EMD filters provide a better perfor mance than the FIR filter, and serve for the pre processing of the envelope signal. Furthermore, because of the quick implementation of the wavelet filtering over the EMD method, this filter can be used for signal conditioning for monitoring purposes.

Under certain operational conditions involving soft and small particles, the low frequency information with regard to fluidized bed dynamics can not be recovered from the envelope of the measured signal. This fact can be attributed to both poor signal emissions and measurement construction. 


\section{Nomenclature}

\section{Abbreviations}

EMD Empirical Mode Decomposition

FB Fluidized bed

FIR Finite Impulse Response

ID Inner Diameter

IIR Infinite Impulse Response

IMF Intrinsec Mode Function

PSD Power Spectral Density

THR Threshold

Greek letters

$\omega_{k}(t) \quad$ Instantaneous frequency of IMF $\mathrm{k}(\mathrm{rad} / \mathrm{s})$

$\bar{\omega}_{k} \quad$ Averaged instantaneous frequency of IMF k ( rad/s)

$\rho_{p} \quad$ Particle density $\left(\mathrm{kg} / \mathrm{m}^{3}\right)$

$\sigma \quad$ Noise level (g)

$\varphi_{k}(t) \quad$ Instantaneous phase angle of IMF $\mathrm{k}(\mathrm{rad})$

Upper case letters

$\mathrm{A}(\mathrm{t}) \quad$ Envelope process of the acceleromery signal $(\mathrm{g})$

$\mathrm{C}_{\mathrm{k}}(\mathrm{t}) \quad$ Intrinsec mode function $\mathrm{k}$

D $\quad$ Bed diameter $(\mathrm{m})$

$\mathrm{H} \quad$ Hurst exponent ( )

$\mathrm{H}_{\mathrm{mf}} \quad$ Minimum fluidization height (m)

$\mathrm{K}_{\mathrm{ML}} \quad$ Kolmogorov entropy (bit/s)

M Number of IMF of the decomposed signal

$\mathrm{N} \quad$ Length of the time series

$\mathrm{S}_{\mathrm{H}}(\mathrm{f}) \quad$ Power spectral density of the power law noise $\left(\mathrm{g}^{2} / \mathrm{Hz}\right)$

Lower case letters

$\mathrm{a}(\mathrm{t}) \quad$ Accelerometry signal $(\mathrm{g})$

$\mathrm{b}(\mathrm{t}) \quad$ Hilbert Transform of $\mathrm{a}(\mathrm{t})(\mathrm{g})$

$\mathrm{d}_{\mathrm{p}} \quad$ Particle size $(\mathrm{m})$

e(n) Standard Gaussian white noise (independent and identically distributed)

f Frequency $(\mathrm{Hz})$

$\mathrm{f}(\mathrm{n}) \quad$ Clean digital signal

$\mathrm{f}_{\mathrm{s}} \quad$ Sampling frequency $(\mathrm{Hz})$

$\mathrm{t} \quad$ Time $(\mathrm{s})$

$\mathrm{r}(\mathrm{t}) \quad$ Residual

$\mathrm{u}_{0} \quad$ Superficial air velocity $(\mathrm{m} / \mathrm{s})$

$\mathrm{u}_{\mathrm{r}} \quad$ Reduced air velocity $\left(\mathrm{u}_{\mathrm{o}} / \mathrm{u}_{\mathrm{mf}}\right)($ )

$\mathrm{u}_{\mathrm{mf}} \quad$ Miminum fluidization air velocity $(\mathrm{m} / \mathrm{s})$

$\mathrm{x}(\mathrm{n}) \quad$ Noisy digital signal

\section{Acknowledgements}

The authors would like to especially thank Prof. María C. Palancar for her contribution to this work and the useful guiding during those years. Moreover, the financial support from the Spanish Ministry of Research, project CTQ2006 15525 C02 01 is kindly acknowledged.

\section{Appendix A}

When the envelope of the accelerometry signal is extracted by means of the Hilbert transform (Eq. (1)), the low frequencies related with the bulk and bubble dynamics are contaminated by noise (Fig. A.1).

In this paper this noise is removed with low pass FIR, wavelet and EMD filters. Both FIR and EMD filters need a cut off frequency to be implemented. For pressure signals it is well known that a cut off of $20 \mathrm{~Hz}$ let to ensure that all the effects due to large bubbles are taken into account $[10,34]$. Nevertheless, in the case of accelerometry signals, the useful frequency band is not known yet and the cut off

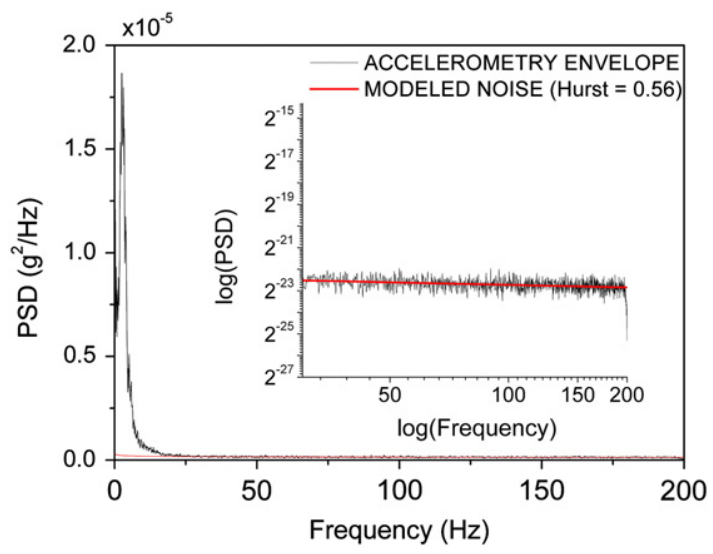

Fig. A.1. Broad band noise present in the envelope. $\mathrm{f}_{\mathrm{s}}=400 \mathrm{~Hz}$.

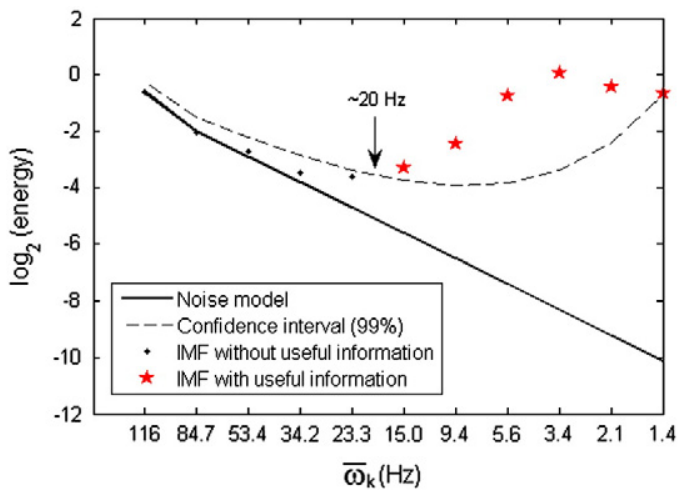

Fig. A.2. Energy of the IMFs for ballotini solid. Each point in the graph correspond to an IMF, the abscissas shows the average instantaneous frequency for each IMF.

frequency must be optimized. With this purpose, the noise present in the Nyquist band $\left(\begin{array}{ll}0 & 200 \mathrm{~Hz}\end{array}\right)$ will be modelled as power law noise [48], since its spectrum can be fitted to Eq. (A.1), [49].

$S_{H}(f)=C|f|^{12 H}$

where $\mathrm{H}$ is referred to as the Hurst exponent and $\mathrm{C}$ is a constant. This model can be solved by fitting the spectrum of the envelope in the range 30 and $200 \mathrm{~Hz}$ (band without useful information in principle) to Eq. (A.1), resulting in Hurst exponents different from 0.5 (colored noise). Once the noise has been modelled, in order to set the cut off frequency the methodology described by Flandrin et al. [50,51] and $\mathrm{Wu}$ and Huang [52] is followed. In this methodology, the signal is decomposed in IMFs and a statistical analysis is carried on over each IMF in order to see if it has useful information or primary noise. According to that, the envelopes were decomposed in IMFs, and their energy was compared with the theoretical energy of the noise. An example is shown in Fig. A.2.

\section{References}

[1] C.S. Daw, W.F. Lawkins, D.J. Downing, N.E. Clapp, Chaotic characteristics of a complex gas-solids flow, Phys. Rev. A 41 (1990) 1179-1181.

[2] J.C. Schouten, C.M. van den Bleek, Chaotic hydrodynamics of fluidization: consequences for scaling and modelling of fluid bed reactors, AlChE Symp. Ser. 88 (1992) 70-84.

[3] J.R. van Ommen, M.O. Coppens, C.M. van den Bleek, J.C. Schouten, Early warning of agglomeration in fluidized beds by attractor comparison, AlChE J. 46 (2000) 2183-2197.

[4] J.R. van Ommen, R.J. de Korte, C.M. van den Bleek, Rapid detection of defluidization using the standard deviation of pressure fluctuations, Chem. Eng. Process. 43 (2004) 1329-1335.

[5] J. Werther, Measurement techniques in fluidized beds, Powder Technol. 102 (1999) 15-36. 
[6] J.G. Yates, S.J.R. Simons, Experimental methods in fluidization research, Int. J. Multiph. Flow 20 (1994) 297-330.

[7] F. Johnsson, R.C. Zijerveld, J.C. Schouten, C.M. van den Bleek, B. Leckner, Characterization of fluidization regimes by time-series analysis of pressure fluctuations, Int. J. Multiph. Flow 26 (2000) 663-715.

[8] S. Sasic, B. Leckner, F. Johnsson, Characterization of fluid dynamics of fluidized beds by analysis of pressure fluctuations, Prog. Energy Combust. Sci. 33 (2007) 453-496

[9] G.D. Cody, D.J. Goldfarb, G.V. Storch, A.N. Norris, Particle granular temperature in gas fluidized beds, Powder Technol. 87 (1996) 211-232.

[10] C.E.A. Finney, C.S. Daw, J.S. Halow, Measuring slugging bed dynamics with acoustic sensors, KONA: Powder Part. 16 (1998) 125-135.

[11] H. Tsujimoto, T. Yokoyama, C.C. Huang, I. Sekiguchi, Monitoring particle fluidization in a fluidized bed granulator with an acoustic emission sensor, Powder Technol. 113 (2000) 88-96.

[12] J.W.R. Boyd, J. Varley, The uses of passive measurement of acoustic emissions from chemical engineering processes, Chem. Eng. Sci. 56 (2001) 1749-1767.

[13] J.W.R. Boyd, J. Varley, Measurement of gas solid hold-up in bubble columns from low frequency acoustic emissions, Chem. Eng. J. 88 (2002) 111-118.

[14] A.H.G. Cents, D.W.F. Brilman, G.F. Versteeg, P.J. Wijnstra, P.P.L. Regtien, Measuring bubble, drop and particle sizes in multiphase systems with ultrasound, AlChE J. 50 (2004) 2750-2762.

[15] J.V. Briongos, J.M. Aragón, M.C. Palancar, Fluidised bed dynamics diagnosis from measurements of low-frequency out-bed passive acoustic emissions, Powder Technol. 162 (2006) 145-156.

[16] D. Vervloet, J. Nijenhuis, J.R. van Ommen, Monitoring a lab-scale fluidized bed dryer: A comparison between pressure transducers, passive acoustic emissions and vibration measurements, Powder Technol. 197 (2010) 36-48.

[17] L. de Martín, J.V. Briongos, J.M. Aragón, M.C. Palancar, Can low frequency accelerometry replace pressure measurements for monitoring gas-solid fluidized beds? Chem. Eng. Sci. 65 (2010) 4055-4064.

[18] R.C. Zijerveld, F. Johnsson, A. Marzocchella, J.C. Schouten, C.M. van den Bleek, Fluidization regimes and transitions from fixed bed to dilute transport flow, Powder Technol. 95 (1998) 185-204.

[19] L. de Martín, Diagnosis of gas-solid fluidized beds by accelerometry, Ph.D. thesis, Complutense University of Madrid, Spain, 2010.

[20] N.C. Nigam, Introduction to random vibrations, The MIT Press, 1983.

[21] R.S. Langley, On various definitions of the envelope of a random process, J. Sound Vib. 105 (1986) 503-512.

[22] L.D. Lutes, S. Sarkani, Random Vibrations: Analysis of Structural and Mechanical Systems, 1st edition, Butterworth-Heinemann, 2003.

[23] D.E. Newland, Introduction to random vibrations, spectral and wavelet analysis, 3rd edition, Dover Publications, 2005.

[24] J. Dugundji, Envelopes and pre-envelopes of real waveforms, IRE Trans. Inf. Theory 4 (1958) 53-57.

[25] C.M. Harris, A.G. Piersol, Harris' Shock and Vibration Handbook, 5th edition, McGraw-Hill, 2002.

[26] S. Satija, L.S. Fan, Characteristics of slugging regime and transition to turbulent regime for fluidized beds of large particles, AlChE J. 31 (1985) 1554-1562.

[27] H. Kage, N. Iwasaki, H. Yamaguchi, Y. Matsuno, Frequency analysis of pressure fluctuation in fluidized bed plenum, J. Chem. Eng. Jpn 24 (1991) 76-81.

[28] J.G. Sun, M.M. Chen, B.T. Chao, Modeling of solids global fluctuations in bubbling fluidized beds by standing surface waves, Int. J. Multiph. Flow 20 (1994) 315-338.

[29] R.C. Brown, E. Brue, Resolving dynamical features of fluidized beds from pressure fluctuations, Powder Technol. 119 (2001) 68-80.
[30] P. Grassberger, I. Procaccia, Estimation of kolmogorov entropy from a chaotic signal, Phys. Rev. A 28 (1983) 2591-2593.

[31] J.C. Schouten, F. Takens, C.M. van den Bleek, Maximum likelihood estimation of the entropy of an attractor, Phys. Rev. E 49 (1994) 126-129.

[32] J. van der Schaaf, J.R. van Ommen, F. Takens, J.C. Schouten, C.M. van den Bleek, Similarity between chaos analysis and frequency analysis of pressure fluctuations in fluidized beds, Chem. Eng. Sci. 59 (2004) 1829-1840.

[33] J.C. Schouten, F. Takens, C.M. van den Bleek, Estimation of the dimension of a noisy attractor, Phys. Rev. E 50 (1994) 1851-1861.

[34] H.M. Letzel, J.C. Schouten, R. Krishna, C.M. van den Bleek, Characterization of regimes and regime transitions in bubble columns by chaos analysis of pressure signals, Chem. Eng. Sci. 52 (1997) 4447-4459.

[35] D.S. Broomhead, J.P. Huke, M.R. Muldoon, Linear filters and non-linear systems, J. R. Soc. Stat. B 54 (1992) 373-382.

[36] T.W. Parks, J.H. McClellan, Chebyshev approximation of nonrecursive digital filters with linear phase, IEEE Trans. Circuit Theory CT-19 (1972) 189-194.

[37] M. Misiti, Y. Misiti, G. Oppenheim, J. Poggi, Wavelet Toolbox. User's Guide, 1996.

[38] I. Daubechies, Ten lectures on wavelets, Society for Industrial and Applied Mathematics, 1992.

[39] D.L. Donoho, J.M. Johnstone, Ideal spatial adaptation by wavelet shrinkage, Biometrika 81 (1994) 425-455.

[40] N.E. Huang Z. Shen, S.R. Long, M.C. Wu, H.H. Shih, Q. Zheng, N.C. Yen, C.C. Tung, H.H. Liu, The empirical mode decomposition and the hilbert spectrum for nonlinear and non-stationary time series analysis, Proc. R. Soc. Lond. A 454 (1998) 903-995.

[41] G. Rilling, P. Flandrin, P. Gonçalvès, On empirical mode decomposition and its algorithms, $6^{\text {th }}$ IEEE/EURASIP Workshop on Nonlinear Signal and Image Processing, Italy, 2003.

[42] J.V. Briongos, J.M. Aragón, M.C. Palancar, Improving non-intrusive measurements techniques for monitoring fluidized bed dynamics, $19^{\text {th }}$ FBC Conference, Austria, 2006.

[43] J. Corella, J.M. Toledo, G. Molina, Calculation of the conditions to get less than $2 \mathrm{~g}$ $\operatorname{tar} / \mathrm{m}^{3} \mathrm{n}$ in a fluidized bed biomass gasifier, Fuel Process. Technol. 87 (2006) 841-846.

[44] V. Skoulou, G. Koufodimos, Z. Samaras, A. Zabaniotou, Low temperature gasification of olive kernels in a 5 -kw fluidized bed reactor for $\mathrm{H}_{2}$-rich producer gas, Int. J. Hydrogen Energy 33 (2008) 6515-6524.

[45] G.F. Hewitt, S. Jayanti, To churn or not to churn, Int. J. Multiph. Flow 19 (1993) 527-529.

[46] M.D. Liébanes, Ciencia y tecnología del secado del alperujo, Ph.D. thesis, University Complutense of Madrid, 2005.

[47] A.P. Baskakov, V.P. Tuponogov, N.F. Filipovsky, A study of pressure fluctuations in a bubbling fluidized bed, Powder Technol. 45 (1986) 113-117.

[48] B. Mandelbrot, Some noises with $\mathrm{l} / \mathrm{f}$ spectrum, a bridge between direct current and white noise, IEEE Trans. Inf. Theory 13 (1967) 289-298.

[49] G. Rilling, P. Flandrin, P. Gonçalvès, Empirical mode decomposition, fractional gaussian noise and hurst exponent estimation, Acoust. Speech Signal Process. 4 (2005) 489-492.

[50] P. Flandrin, G. Rilling, P. Gonçalvès, Empirical mode decomposition as a filter bank, IEEE Signal Process Lett. 11 (2004) 112-114.

[51] P. Flandrin, P. Gonçalvès, G. Rilling, Hilbert-Huang transform and its applications, in chapter: EMD equivalent filter banks, from interpretation to applications, Word Scientific Publishing Company, 2005, pp. 57-73.

[52] X. Wu, N.E. Huang, A study of the characteristics of white noise using the empirical mode decomposition method, Proc. R. Soc. Lond. A 460 (2004) 1597-1611. 